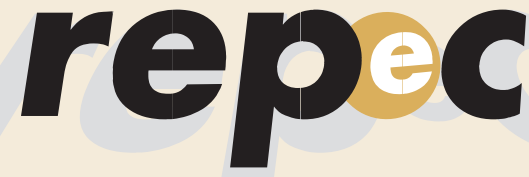

REPeC, Brasília, v. 8, n. 4, informações editoriais, p. 464-473, out./dez. 2014

Disponível online em www.repec.org.br
Revista de Educação e Pesquisa em Contabilidade Journal of Education and Research in Accounting Revista de Educación e Investigación en Contabilidad

Periódico Trimestral, digital e gratuito publicado pela Academia Brasileira de Ciências Contábeis

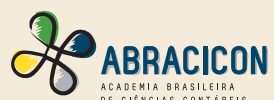

ISSN 1981-8610

\title{
Informações Editoriais 2014
}

\section{Artigos Publicados - 2014}

\begin{tabular}{lcc}
\hline Edições & Autores por Edição & Artigos por Edição \\
\hline Jan./Mar., 2014 & 16 & 6 \\
\hline Abr./Jun., 2014 & 20 & 6 \\
\hline Jul./Set., 2014 & 17 & 6 \\
\hline Out./Dez., 2014 & 17 & 6 \\
\hline Total & 70 & 24 \\
\hline Média & 17,5 & 6 \\
\hline
\end{tabular}

Número de Artigos por Unidade de Federação - 2014

\begin{tabular}{|c|c|c|c|c|c|c|c|c|c|c|}
\hline \multirow{3}{*}{ Unidade da Federação } & \multicolumn{10}{|c|}{ Edições } \\
\hline & \multicolumn{2}{|c|}{ Jan./Mar. } & \multicolumn{2}{|c|}{ Abr./Jun. } & \multicolumn{2}{|c|}{ Jul./Set. } & \multicolumn{2}{|c|}{ Out./Dez. } & \multicolumn{2}{|c|}{ Total } \\
\hline & $\mathrm{Fi}$ & $\mathrm{Fi} \%$ & $\mathrm{Fi}$ & $\mathrm{Fi} \%$ & $\mathrm{Fi}$ & $\mathrm{Fi} \%$ & $\mathrm{Fi}$ & $\mathrm{Fi} \%$ & $\mathrm{Fi}$ & $\mathrm{Fi} \%$ \\
\hline BA & - & $0 \%$ & 1 & $17 \%$ & - & $0 \%$ & - & $0 \%$ & 1 & $4 \%$ \\
\hline $\mathrm{BA} / \mathrm{SP}$ & 1 & $17 \%$ & - & $0 \%$ & - & $0 \%$ & - & $0 \%$ & 1 & $4 \%$ \\
\hline CE & - & $0 \%$ & - & $0 \%$ & - & $0 \%$ & 1 & $17 \%$ & 1 & $4 \%$ \\
\hline ES & - & $0 \%$ & - & $0 \%$ & - & $0 \%$ & 1 & $17 \%$ & 1 & $4 \%$ \\
\hline$E S / R J$ & - & $0 \%$ & - & $0 \%$ & 1 & $17 \%$ & - & $0 \%$ & 1 & $4 \%$ \\
\hline MG/SP & 1 & $17 \%$ & 1 & $17 \%$ & - & $0 \%$ & - & $0 \%$ & 2 & $8 \%$ \\
\hline DF/RN & - & $0 \%$ & - & $0 \%$ & - & $0 \%$ & 1 & $17 \%$ & 1 & $4 \%$ \\
\hline DF & - & $0 \%$ & 1 & $17 \%$ & - & $0 \%$ & & $0 \%$ & 1 & $4 \%$ \\
\hline PR & - & $0 \%$ & 1 & $17 \%$ & - & $0 \%$ & 2 & $33 \%$ & 3 & $13 \%$ \\
\hline PR/RS & - & $0 \%$ & - & $0 \%$ & 1 & $17 \%$ & - & $0 \%$ & 1 & $4 \%$ \\
\hline $\mathrm{PI}$ & 1 & $17 \%$ & - & $0 \%$ & - & $0 \%$ & - & $0 \%$ & 1 & $4 \%$ \\
\hline $\mathrm{RJ}$ & - & $0 \%$ & 1 & $17 \%$ & - & $0 \%$ & - & $0 \%$ & 1 & $4 \%$ \\
\hline RS & 1 & $17 \%$ & - & $0 \%$ & - & $0 \%$ & - & $0 \%$ & 1 & $4 \%$ \\
\hline SC & - & $0 \%$ & 1 & $17 \%$ & - & $0 \%$ & 1 & $17 \%$ & 2 & $8 \%$ \\
\hline SC/PR & - & $0 \%$ & - & $0 \%$ & 1 & $17 \%$ & - & $0 \%$ & 1 & $4 \%$ \\
\hline SP & 1 & $17 \%$ & - & $0 \%$ & 3 & $50 \%$ & - & $0 \%$ & 4 & $17 \%$ \\
\hline Portugal & 1 & $17 \%$ & - & $0 \%$ & - & $0 \%$ & - & $0 \%$ & 1 & $4 \%$ \\
\hline Total & 6 & $100 \%$ & 6 & $100 \%$ & 6 & $100 \%$ & 6 & $100 \%$ & 24 & $100 \%$ \\
\hline
\end{tabular}


Número de Autores por Unidade de Federação - 2014

\begin{tabular}{|c|c|c|c|c|c|c|c|c|c|c|}
\hline \multirow{3}{*}{ Unidade da Federação } & \multicolumn{10}{|c|}{ Edições } \\
\hline & \multicolumn{2}{|c|}{ Jan./Mar. } & \multicolumn{2}{|c|}{ Abr./Jun. } & \multicolumn{2}{|c|}{ Jul./Set. } & \multicolumn{2}{|c|}{ Out./Dez. } & \multicolumn{2}{|c|}{ Total } \\
\hline & $\mathrm{Fi}$ & $\mathrm{Fi} \%$ & $\mathrm{Fi}$ & $\mathrm{Fi} \%$ & $\mathrm{Fi}$ & $\mathrm{Fi} \%$ & $\mathrm{Fi}$ & $\mathrm{Fi} \%$ & $\mathrm{Fi}$ & $\mathrm{Fi} \%$ \\
\hline Bahia & 2 & $12 \%$ & 5 & $29 \%$ & - & $0 \%$ & - & $0 \%$ & 7 & $10 \%$ \\
\hline Ceará & - & $0 \%$ & - & $0 \%$ & - & $0 \%$ & 2 & $12 \%$ & 2 & $3 \%$ \\
\hline Distrito Federal & - & $0 \%$ & 3 & $18 \%$ & - & $0 \%$ & 1 & $6 \%$ & 4 & $6 \%$ \\
\hline Espírito Santo & - & $0 \%$ & - & $0 \%$ & 3 & $18 \%$ & 3 & $18 \%$ & 6 & $9 \%$ \\
\hline Minas Gerais & 3 & $18 \%$ & 1 & $6 \%$ & - & $0 \%$ & - & $0 \%$ & 4 & $6 \%$ \\
\hline Paraná & - & $0 \%$ & 4 & $24 \%$ & 2 & $12 \%$ & 6 & $35 \%$ & 12 & $17 \%$ \\
\hline Piauí & 2 & $12 \%$ & - & $0 \%$ & - & $0 \%$ & - & $0 \%$ & 2 & $3 \%$ \\
\hline Rio de Janeiro & - & $0 \%$ & 3 & $18 \%$ & 1 & $6 \%$ & - & $0 \%$ & 4 & $6 \%$ \\
\hline Rio Grande do Sul & 2 & $12 \%$ & - & $0 \%$ & 2 & $12 \%$ & - & $0 \%$ & 4 & $6 \%$ \\
\hline Rio Grande do Norte & - & $0 \%$ & - & $0 \%$ & - & $0 \%$ & 1 & $6 \%$ & 1 & $1 \%$ \\
\hline Santa Catarina & - & $0 \%$ & 2 & $12 \%$ & 1 & $6 \%$ & 4 & $24 \%$ & 7 & $10 \%$ \\
\hline São Paulo & 4 & $24 \%$ & 2 & $12 \%$ & 8 & $47 \%$ & - & $0 \%$ & 14 & $20 \%$ \\
\hline Portugal & 3 & $18 \%$ & - & $0 \%$ & - & $0 \%$ & - & $0 \%$ & 3 & $4 \%$ \\
\hline Total & 16 & $100 \%$ & 20 & $100 \%$ & 17 & $100 \%$ & 17 & $100 \%$ & 70 & $100 \%$ \\
\hline
\end{tabular}

\section{Inventário dos Artigos - 2014}

\begin{tabular}{lc}
\hline Itens & 2014 \\
\hline $\begin{array}{l}\text { Estoque inicial de artigos em 10 de janeiro } \\
\text { Em avaliação } \\
\text { Aprovados }\end{array}$ & 24 \\
\hline Número de artigos submetidos & 1 \\
\hline Número de artigos não aprovados no Desk Review & 107 \\
\hline Número de artigos reprovados & $(28)$ \\
\hline Número de artigos publicados & $(55)$ \\
\hline Estoque Final de artigos em avaliação em 31 de dezembro & $(24)$ \\
\hline
\end{tabular}


Instituições de Origem dos Autores dos Artigos - 2014

\begin{tabular}{|c|c|c|c|c|c|c|}
\hline \multirow{3}{*}{$\begin{array}{l}\text { UF/IES de origem } \\
\text { BA/UESB }\end{array}$} & \multicolumn{6}{|c|}{ Edições } \\
\hline & \multirow{2}{*}{$\frac{\text { Jan./Mar. }}{2}$} & \multirow[t]{2}{*}{ Abr./Jun. } & \multirow[t]{2}{*}{ Jul./Set. } & \multirow[t]{2}{*}{ Out./Dez. } & \multicolumn{2}{|c|}{ Total } \\
\hline & & & & & 2 & $3 \%$ \\
\hline BA/UFBA & & 5 & & & 5 & $7 \%$ \\
\hline CE/UFC & & & & 1 & 1 & $1 \%$ \\
\hline CE/UNIFOR & & & & 1 & 1 & $1 \%$ \\
\hline DF/UNB & & 3 & & 1 & 4 & $6 \%$ \\
\hline ES/FUCAPE & & & 3 & & 3 & $4 \%$ \\
\hline ES/UFES & & & & 3 & 3 & $4 \%$ \\
\hline MG/UFMG & 3 & & & & 3 & $4 \%$ \\
\hline MG/UFU & & 1 & & & 1 & $1 \%$ \\
\hline PR/UFPR & & 4 & 2 & & 6 & $9 \%$ \\
\hline PR/UEM & & & & 5 & 5 & $7 \%$ \\
\hline PR/UNIBRASIL & & & & 1 & 1 & $1 \%$ \\
\hline $\mathrm{PI} / \mathrm{UFPI}$ & 2 & & & & 2 & $3 \%$ \\
\hline RJ/UFRJ & & 3 & & & 3 & $4 \%$ \\
\hline RJ/UFF & & & 1 & & 1 & $1 \%$ \\
\hline RN/UFRN & & & & 1 & 1 & $1 \%$ \\
\hline RS/UNISINOS & 2 & & 2 & & 4 & $6 \%$ \\
\hline SC/UFSC & & 2 & & 1 & 3 & $4 \%$ \\
\hline SC/FURB & & & 1 & 1 & 2 & $3 \%$ \\
\hline SC/UDESC & & & & 2 & 2 & $3 \%$ \\
\hline SP/UNIP & 1 & & & & 1 & $1 \%$ \\
\hline SP/USP & 2 & 2 & 3 & & 7 & $10 \%$ \\
\hline SP/USPRP & 1 & & & & 1 & $1 \%$ \\
\hline SP/UPM & & & 3 & & 3 & $4 \%$ \\
\hline SP/FGV & & & 1 & & 1 & $1 \%$ \\
\hline SP/UNINOVE & & & 1 & & 1 & $1 \%$ \\
\hline Portugal/UV & 3 & & & & 3 & $4 \%$ \\
\hline Total & 16 & 20 & 17 & 17 & 70 & $100 \%$ \\
\hline
\end{tabular}




\section{Periodo Médio de Circulação dos Artigos Publicados - 2014}

Vol. 8, n. 1, 2014

\begin{tabular}{|c|c|c|c|c|c|c|c|c|c|}
\hline $\begin{array}{l}\mathrm{N}^{\circ} \text {. do } \\
\text { Artigo }\end{array}$ & $\begin{array}{c}\text { Data de } \\
\text { submissão }\end{array}$ & $\begin{array}{l}\text { Avaliado } \\
\text { pelos } \\
\text { Pares }\end{array}$ & $\begin{array}{l}\text { Reformulado } \\
\text { pelos autores }\end{array}$ & $\begin{array}{c}\text { Data da } \\
\text { Aceitação }\end{array}$ & $\begin{array}{c}\text { Data da } \\
\text { Publicação }\end{array}$ & $\begin{array}{c}N^{\circ} \text {. de } \\
\text { dias para } \\
\text { avaliação }\end{array}$ & $\begin{array}{c}\mathrm{N}^{\circ} \text {. de } \\
\text { dias para } \\
\text { reformulação }\end{array}$ & $\begin{array}{c}\mathbf{N}^{\circ} \text {. de } \\
\text { dias para } \\
\text { recomendação } \\
\text { de publicação }\end{array}$ & $\begin{array}{c}\mathrm{N}^{\circ} \text {. de dias } \\
\text { entre a } \\
\text { submissão e } \\
\text { publicação }\end{array}$ \\
\hline 1 & $16 / 09 / 2013$ & $24 / 10 / 2013$ & $10 / 11 / 2013$ & $19 / 11 / 2013$ & $31 / 03 / 2014$ & 38 & 16 & 9 & 195 \\
\hline 2 & $19 / 09 / 2013$ & $06 / 12 / 2013$ & $11 / 01 / 2014$ & $11 / 01 / 2014$ & $31 / 03 / 2014$ & 77 & 35 & 0 & 192 \\
\hline 3 & $12 / 03 / 2013$ & $22 / 08 / 2013$ & 05/11/2013 & $16 / 01 / 2014$ & $31 / 03 / 2014$ & 160 & 73 & 71 & 379 \\
\hline 4 & $25 / 02 / 2013$ & $19 / 11 / 2013$ & $11 / 12 / 2013$ & 08/01/2014 & $31 / 03 / 2014$ & 264 & 22 & 27 & 396 \\
\hline 5 & $23 / 08 / 2013$ & $10 / 10 / 2013$ & $28 / 12 / 2013$ & 06/01/2014 & $31 / 03 / 2014$ & 47 & 78 & 8 & 218 \\
\hline 6 & $11 / 07 / 2013$ & $12 / 09 / 2013$ & $13 / 12 / 2013$ & $30 / 12 / 2013$ & $31 / 03 / 2014$ & 61 & 91 & 17 & 260 \\
\hline \multicolumn{6}{|c|}{ Média de dias para publicação de artigos por Edição } & 108 & & & 273 \\
\hline \multicolumn{6}{|c|}{ Média de meses para publicação de artigos por Edição } & 3,6 & & & 9,1 \\
\hline
\end{tabular}

Vol. 8, n. 2, 2014

\begin{tabular}{|c|c|c|c|c|c|c|c|c|c|}
\hline $\begin{array}{l}\mathrm{N}^{\circ} \text {. do } \\
\text { Artigo }\end{array}$ & $\begin{array}{c}\text { Data de } \\
\text { submissão }\end{array}$ & $\begin{array}{l}\text { Avaliado } \\
\text { pelos } \\
\text { Pares }\end{array}$ & $\begin{array}{l}\text { Reformulado } \\
\text { pelos autores }\end{array}$ & $\begin{array}{c}\text { Data da } \\
\text { Aceitação }\end{array}$ & $\begin{array}{c}\text { Data da } \\
\text { Publicação }\end{array}$ & $\begin{array}{c}N^{\circ} \text {. de } \\
\text { dias para } \\
\text { avaliação }\end{array}$ & $\begin{array}{c}N^{\circ} \text {. de } \\
\text { dias para } \\
\text { reformulação }\end{array}$ & $\begin{array}{c}\mathrm{N}^{\circ} \text {. de } \\
\text { dias para } \\
\text { recomendação } \\
\text { de publicação }\end{array}$ & $\begin{array}{c}\mathrm{N}^{\circ} \text {. de dias } \\
\text { entre a } \\
\text { submissão e } \\
\text { publicação }\end{array}$ \\
\hline 1 & 09/10/2013 & 06/01/2014 & 20/01/2014 & $20 / 02 / 2014$ & $30 / 06 / 2014$ & 87 & 14 & 30 & 261 \\
\hline 2 & $14 / 09 / 2013$ & 21/03/2014 & 28/03/2014 & 09/04/2014 & $30 / 06 / 2014$ & 187 & 7 & 11 & 286 \\
\hline 3 & 09/10/2013 & 06/05/2014 & $19 / 05 / 2014$ & $19 / 05 / 2014$ & $30 / 06 / 2014$ & 207 & 13 & 0 & 261 \\
\hline 4 & $17 / 10 / 2013$ & $01 / 02 / 2014$ & $13 / 03 / 2014$ & $17 / 03 / 2014$ & $30 / 06 / 2014$ & 104 & 42 & 4 & 253 \\
\hline 5 & $25 / 07 / 2013$ & $30 / 12 / 2013$ & $27 / 01 / 2014$ & $16 / 04 / 2014$ & $30 / 06 / 2014$ & 155 & 27 & 79 & 335 \\
\hline 6 & 25/09/2013 & $31 / 12 / 2013$ & $22 / 02 / 2014$ & $17 / 03 / 2014$ & $30 / 06 / 2014$ & 96 & 52 & 25 & 275 \\
\hline \multicolumn{6}{|c|}{ Média de dias para publicação de artigos por Edição } & 139 & & & 279 \\
\hline \multicolumn{6}{|c|}{ Média de meses para publicação de artigos por Edição } & 4,6 & & & 9,3 \\
\hline
\end{tabular}


Vol. 8, n. 3, 2014

\begin{tabular}{|c|c|c|c|c|c|c|c|c|c|}
\hline $\begin{array}{l}N^{\circ} \text {. do } \\
\text { Artigo }\end{array}$ & $\begin{array}{c}\text { Data de } \\
\text { submissão }\end{array}$ & $\begin{array}{l}\text { Avaliado } \\
\text { pelos } \\
\text { Pares }\end{array}$ & $\begin{array}{l}\text { Reformulado } \\
\text { pelos autores }\end{array}$ & $\begin{array}{c}\text { Data da } \\
\text { Aceitação }\end{array}$ & $\begin{array}{c}\text { Data da } \\
\text { Publicação }\end{array}$ & $\begin{array}{c}N^{\circ} \text {. de } \\
\text { dias para } \\
\text { avaliação }\end{array}$ & $\begin{array}{c}\mathrm{N}^{\circ} \text {. de } \\
\text { dias para } \\
\text { reformulação }\end{array}$ & $\begin{array}{c}\mathrm{N}^{\circ} \text {. de } \\
\text { dias para } \\
\text { recomendação } \\
\text { de publicação }\end{array}$ & $\begin{array}{c}\mathrm{N}^{\circ} \text {. de dias } \\
\text { entre a } \\
\text { submissão e } \\
\text { publicação }\end{array}$ \\
\hline 1 & 05/08/2013 & $04 / 06 / 2014$ & $10 / 06 / 2014$ & $13 / 06 / 2014$ & $30 / 09 / 2014$ & 299 & 6 & 3 & 415 \\
\hline 2 & 23/10/2013 & $16 / 06 / 2014$ & $22 / 06 / 2014$ & $30 / 06 / 2014$ & $30 / 09 / 2014$ & 233 & 6 & 8 & 337 \\
\hline 3 & $20 / 04 / 2014$ & $24 / 07 / 2014$ & 28/07/2014 & 29/07/2014 & $30 / 09 / 2014$ & 94 & 4 & 1 & 160 \\
\hline 4 & $07 / 11 / 2013$ & 08/03/2014 & $22 / 05 / 2014$ & 29/07/2014 & $30 / 09 / 2014$ & 121 & 74 & 67 & 323 \\
\hline 5 & 06/03/2014 & $30 / 06 / 2014$ & 29/07/2014 & $30 / 07 / 2014$ & $30 / 09 / 2014$ & 114 & 29 & 1 & 204 \\
\hline 6 & 09/02/2014 & $19 / 07 / 2014$ & 29/07/2014 & 29/07/2014 & $30 / 09 / 2014$ & 160 & 10 & 0 & 231 \\
\hline \multicolumn{5}{|c|}{ Média de dias para publicação de artigos por Edição } & & 170 & & & 278 \\
\hline \multicolumn{6}{|c|}{ Média de meses para publicação de artigos por Edição } & 5,7 & & & 9,3 \\
\hline
\end{tabular}

Vol. 8, n. 4, 2014

\begin{tabular}{|c|c|c|c|c|c|c|c|c|c|}
\hline $\begin{array}{l}N^{\circ} \text {. do } \\
\text { Artigo }\end{array}$ & $\begin{array}{c}\text { Data de } \\
\text { submissão }\end{array}$ & $\begin{array}{l}\text { Avaliado } \\
\text { pelos } \\
\text { Pares }\end{array}$ & $\begin{array}{l}\text { Reformulado } \\
\text { pelos autores }\end{array}$ & $\begin{array}{c}\text { Data da } \\
\text { Aceitação }\end{array}$ & $\begin{array}{c}\text { Data da } \\
\text { Publicação }\end{array}$ & $\begin{array}{c}N^{\circ} \text {. de } \\
\text { dias para } \\
\text { avaliação }\end{array}$ & $\begin{array}{c}N^{\circ} \text {. de } \\
\text { dias para } \\
\text { reformulação }\end{array}$ & $\begin{array}{c}\mathrm{N}^{\circ} \text {. de } \\
\text { dias para } \\
\text { recomendação } \\
\text { de publicação }\end{array}$ & $\begin{array}{c}\mathrm{N}^{\circ} \text {. de dias } \\
\text { entre a } \\
\text { submissão e } \\
\text { publicação }\end{array}$ \\
\hline 1 & $18 / 05 / 2014$ & $18 / 08 / 2014$ & $28 / 08 / 2014$ & 03/10/2014 & $24 / 12 / 2014$ & 90 & 10 & 35 & 216 \\
\hline 2 & 24/10/2013 & $16 / 03 / 2014$ & 24/10/2014 & $02 / 11 / 2014$ & 24/12/2014 & 142 & 218 & 8 & 420 \\
\hline 3 & 07/04/2014 & 29/09/2014 & 19/10/2014 & $01 / 11 / 2014$ & $24 / 12 / 2014$ & 172 & 20 & 12 & 257 \\
\hline 4 & 19/03/2014 & 13/09/2014 & 27/09/2014 & $11 / 10 / 2014$ & 24/12/2014 & 174 & 14 & 14 & 275 \\
\hline 5 & $11 / 10 / 2014$ & 03/11/2014 & 05/11/2014 & $05 / 11 / 2014$ & 24/12/2014 & 22 & 2 & 0 & 73 \\
\hline 6 & $14 / 10 / 2014$ & $18 / 10 / 2014$ & 24/10/2014 & $03 / 11 / 2014$ & 24/12/2014 & 4 & 6 & 9 & 70 \\
\hline \multicolumn{6}{|c|}{ Média de dias para publicação de artigos por Edição } & 101 & & & 219 \\
\hline \multicolumn{6}{|c|}{ Média de meses para publicação de artigos por Edição } & 3,4 & & & 7,3 \\
\hline
\end{tabular}

\begin{tabular}{lcc}
\hline Média de dias para publicação de artigos por Edição em 2014 & 129,5 & 262,2 \\
\hline Média de meses para publicação de artigos por Edição em 2014 & 4,3 & 8,7 \\
\hline
\end{tabular}




\section{Avaliadores Ad Hoc e Instituição de Vínculo - 2014}

\begin{tabular}{|c|c|}
\hline Avaliador & Instituição \\
\hline Adriana Maria Procópio de Araujo & Universidade de São Paulo - Ribeirão Preto \\
\hline Adriano Rodrigues & Universidade Federal do Rio de Janeiro \\
\hline Alessandra Vasconcelos Gallon & Universidade Federal do Ceará \\
\hline Alexandre Reis Rosa & Universidade Federal do Espírito Santo \\
\hline Alexsandro Broedel Lopes & Universidade de São Paulo \\
\hline Alfredo Sarlo Neto & Universidade Federal do Espírito Santo \\
\hline Alvaro Augusto Ricardino Filho & Pontifícia Universidade Católica de São Paulo \\
\hline Alvaro Martim Guedes & Universidade Estadual Paulista “Julio Mesquita Neto" \\
\hline Amaury José Rezende & Universidade de São Paulo - Ribeirão Preto \\
\hline Ana Cristina de Faria & Universidade Municipal de São Caetano do Sul \\
\hline Ana Maria Roux César & Universidade Presbiteriana Mackenzie \\
\hline André Carlos Busaneli De Aquino & Universidade de São Paulo - Ribeirão Preto \\
\hline Andson Braga De Aguiar & Universidade de São Paulo \\
\hline Aneide Oliveira Araujo & Universidade Federal do Rio Grande do Norte \\
\hline Antonia Silva de Lima & Universidade Federal do Amazonas \\
\hline Antonio Artur de Souza & Universidade Federal de Minas Gerais \\
\hline Antonio Carlos Dias Coelho & Universidade Federal do Ceará \\
\hline Antonio Gonçalves de Oliveira & Universidade Tecnológica Federal do Paraná \\
\hline Antonio Lopo Martinez & Fucape Business School \\
\hline Aridelmo José Campanharo Teixeira & Fucape Business School \\
\hline Arilda Magda Campagnaro Teixeira & Fucape Business School \\
\hline Artur Roberto do Nascimento & Universidade Estadual de Feira de Santana \\
\hline Bruno Funchal & Fucape Business School \\
\hline Cacilda Andrade & Universidade Federal de Pernambuco \\
\hline Carlos Alberto Diehl & Universidade do Vale do Rio dos Sinos \\
\hline Carlos Eduardo Facin Lavarda & Fundação Universidade Regional de Blumenau \\
\hline Carlos Renato Theóphilo & Universidade Estadual de Montes Claros \\
\hline César Augusto Tiburcio Silva & Universidade de Brasília \\
\hline Claudio de Souza Miranda & Universidade de São Paulo - Ribeirão Preto \\
\hline Daphins Teodoro da Silva Junior & Universidade de São Paulo \\
\hline Davi Rogerio De Moura Costa & Universidade de São Paulo \\
\hline Deisy Cristina Corrêa Igarashi & Universidade Estadual do Oeste do Paraná \\
\hline Delci Grapégia Dal Vesco & Universidade Estadual do Oeste do Paraná \\
\hline Denizar Leal & Universidade Federal do Espírito Santo \\
\hline Dione Olesczuk Soutes & Universidade Estadual do Oeste do Paraná \\
\hline
\end{tabular}




\begin{tabular}{|c|c|}
\hline Edgard B. Cornachione Jr & Universidade de São Paulo \\
\hline Edilene Santos Santana & Fundação Getúlio Vargas - São Paulo \\
\hline Edílson Paulo & Universidade Federal da Paraíba \\
\hline Elionor Farah Jreige Weffort & Fundação Escola de Comércio Álvares Penteado \\
\hline Eliseu Martins & Universidade de São Paulo \\
\hline Emanuel Junqueira & Universidade Federal do Espírito Santo \\
\hline Eric Aversari Martins & Universidade de São Paulo \\
\hline Ernani Ott & Universidade do Vale do Rio dos Sinos \\
\hline Fábio Frezatti & Universidade de São Paulo \\
\hline Fabio Gallo Garcia & Fundação Getúlio Vargas - São Paulo \\
\hline Fábio Moraes da Costa & Fucape Business School \\
\hline Felipe Ramos Ferreira & Fucape Business School \\
\hline Fernando Caio Galdi & Fucape Business School \\
\hline Fernando Dal-Ri Murcia & Universidade Federal de Santa Catarina \\
\hline Fernando de Almeida Santos & Pontifícia Universidade Católica de São Paulo \\
\hline Flávia Zóboli Dalmácio & Universidade de São Paulo \\
\hline Francisco Antonio Bezerra & Fundação Universidade Regional de Blumenau \\
\hline Gerlando Augusto Sampaio Franco de Lima & Universidade de São Paulo \\
\hline Guillermo Braunbeck & Universidade de São Paulo \\
\hline Henrique Formigoni & Universidade Presbiteriana Mackenzie \\
\hline Ilse Maria Beuren & Universidade Federal do Paraná \\
\hline Irineu Afonso Frey & Universidade Federal de Santa Catarina \\
\hline Jacqueline Veneroso Alves da Cunha & Universidade Federal de Minas Gerais \\
\hline Jaime Crozatti & Universidade de São Paulo \\
\hline Jorge de Souza Bispo & Receita Federal do Brasil \\
\hline Jorge Eduardo Scarpin & Universidade Federal do Paraná \\
\hline Jorge Katsumi Niyama & Universidade de Brasília \\
\hline José Alonso Borba & Universidade Federal de Santa Catarina \\
\hline José Ellias Feres de Almeida & Universidade Federal do Espírito Santo \\
\hline José Maria Dias Filho & Universidade Federal da Bahia \\
\hline Laura Calixto & Universidade Federal de São Paulo \\
\hline Lauro Brito De Almeida & Universidade Federal do Paraná \\
\hline Luciano Gomes Reis & Universidade Estadual de Londrina \\
\hline Luis Eduardo Afonso & Universidade de São Paulo \\
\hline Luiz Alberton & Universidade Federal de Santa Catarina \\
\hline
\end{tabular}




\begin{tabular}{|c|c|}
\hline Maisa de Souza Ribeiro & Universidade de São Paulo - Ribeirão Preto \\
\hline Marcelle Colares Oliveira & Universidade Federal do Ceará \\
\hline Marcelo Alvaro da Silva Macedo & Universidade Federal do Rio de Janeiro \\
\hline Marcelo Sanches Pagliarussi & Universidade de São Paulo - Ribeirão Preto \\
\hline Marcia Maria dos Santos Bortolocci Espejo & Universidade Federal do Paraná \\
\hline Márcia Martins Mendes De Luca & Universidade Federal do Ceará \\
\hline Marcio Luiz Borinelli & Universidade de São Paulo \\
\hline Marcos Antonio de Souza & Universidade do Vale do Rio dos Sinos \\
\hline Marcus Vinicius Veras Machado & Universidade Federal do Ceará \\
\hline Maria José Carvalho de Souza Domingues & Fundação Universidade Regional de Blumenau \\
\hline Maria Thereza Pompa Antunes, Doutora & Universidade de São Paulo \\
\hline Mayla Cristina Costa & Universidade Federal do Paraná \\
\hline Moacir Moacir Sancovschi & Universidade Federal do Rio de Janeiro \\
\hline Monica Sionara Schpallir Calijuri & Universidade Presbiteriana Mackenzie \\
\hline Otávio Ribeiro De Medeiros & Universidade de Brasília \\
\hline Patricia Gonzalez Gonzalez & Universidade del Valle (Colômbia) \\
\hline Patricia Maria Bortolon & Universidade Federal do Espírito santo \\
\hline Paulo Roberto Barbosa Lustosa & Universidade de Brasília \\
\hline Paulo Roberto Da Cunha & Fundação Universidade Regional de Blumenau \\
\hline Paulo Rogério Faustino Matos & Universidade Federal do Ceará \\
\hline Raimundo Nonato Lima Filho & Universidade do Estado da Bahia \\
\hline Reinaldo Guerreiro & Universidade de São Paulo \\
\hline Renê Coppe Pimentel & Fucape Business School \\
\hline Rogério João Lunkes & Universidade Federal de Santa Catarina \\
\hline Romualdo Douglas Colauto & Universidade Federal do Paraná \\
\hline Sérgio De ludícibus & Pontifícia Universidade Católica de São Paulo \\
\hline Silvia Pereira De Castro Casa Nova & Universidade de São Paulo \\
\hline Solange Garcia dos Reis & Universidade de São Paulo - Ribeirão Preto \\
\hline Sonia Maria da Silva Gomes & Universidade Federal da Bahia \\
\hline Umbelina Cravo Texeira Lagioia & Universidade Federal de Pernambuco \\
\hline Valcemiro Nossa & Fucape Business School \\
\hline Vera Maria Rodrigues Ponte & Universidade Federal do Ceará \\
\hline Victor Branco de Holanda & Universidade Federal do Rio Grande do Norte \\
\hline Vinicius Aversari Martins & Universidade de São Paulo - Ribeirão Preto \\
\hline Wagner Moura Lamounier & Universidade Federal de Minas Gerais \\
\hline Wellington Rocha & Universidade de São Paulo \\
\hline
\end{tabular}




\section{Origem dos Avaliadores - 2014}

\begin{tabular}{lcc}
\hline Estado & Quantidade & Percentual \\
\hline Amazonas & 1 & $0,9 \%$ \\
\hline Bahia & 4 & $3,8 \%$ \\
\hline Ceará & 7 & $6,6 \%$ \\
\hline Distrito Federal & 4 & $3,8 \%$ \\
\hline Espirito Santo & 15 & $14,2 \%$ \\
\hline Minas Gerais & 4 & $3,8 \%$ \\
\hline Paraíba & 1 & $0,9 \%$ \\
\hline Pernambuco & 2 & $1,9 \%$ \\
\hline Paraná & 11 & $10,4 \%$ \\
\hline Rio de Janeiro & 3 & $2,8 \%$ \\
\hline Rio Grande do Norte & 2 & $1,9 \%$ \\
\hline Rio Grande do Sul & 3 & $2,8 \%$ \\
\hline Santa Catarina & 9 & $8,5 \%$ \\
\hline São Paulo & 39 & $36,8 \%$ \\
\hline Colômbia & 1 & $0,9 \%$ \\
\hline Total & 106 & $100 \%$ \\
\hline
\end{tabular}


Instituições de Lotação dos Avaliadores - 2014

\begin{tabular}{|c|c|c|c|}
\hline Instituição & Estado & Quantidade & Percentual \\
\hline Universidade Federal do Amazonas & AM & 1 & $0,9 \%$ \\
\hline Universidade Estadual de Feira de Santana & BA & 1 & $0,9 \%$ \\
\hline Universidade Federal da Bahia & BA & 2 & $1,9 \%$ \\
\hline Universidade do Estado da Bahia & BA & 1 & $0,9 \%$ \\
\hline Universidade Federal do Ceará & CE & 7 & $6,6 \%$ \\
\hline Universidade de Brasília & DF & 4 & $3,8 \%$ \\
\hline Fucape Business School & ES & 9 & $8,5 \%$ \\
\hline Universidade Federal do Espírito Santo & ES & 6 & $5,7 \%$ \\
\hline Universidade Estadual de Montes Claros & MG & 1 & $0,9 \%$ \\
\hline Universidade Federal de Minas Gerais & MG & 3 & $2,8 \%$ \\
\hline Universidade Federal da Paraíba & PB & 1 & $0,9 \%$ \\
\hline Universidade Federal de Pernambuco & PE & 2 & $1,9 \%$ \\
\hline Universidade Estadual de Londrina & PR & 1 & $0,9 \%$ \\
\hline Universidade Estadual do Oeste do Paraná & PR & 3 & $2,8 \%$ \\
\hline Universidade Federal do Paraná & PR & 6 & $5,7 \%$ \\
\hline Universidade Tecnológica Federal do Paraná & PR & 1 & $0,9 \%$ \\
\hline Universidade Federal do Rio de Janeiro & RJ & 3 & $2,8 \%$ \\
\hline Universidade Federal do Rio Grande do Norte & RN & 2 & $1,9 \%$ \\
\hline Universidade do Vale do Rio dos Sinos & RS & 3 & $2,8 \%$ \\
\hline Fundação Universidade Regional de Blumenau & SC & 4 & $3,8 \%$ \\
\hline Universidade Federal de Santa Catarina & SC & 5 & $4,7 \%$ \\
\hline Fundação Escola de Comércio Álvares Penteado & $\mathrm{SP}$ & 1 & $0,9 \%$ \\
\hline Fundação Getúlio Vargas - SP & SP & 2 & $1,9 \%$ \\
\hline Pontifícia Universidade Católica de São Paulo & $\mathrm{SP}$ & 3 & $2,8 \%$ \\
\hline Receita Federal do Brasil & SP & 1 & $0,9 \%$ \\
\hline Universidade de São Paulo & SP & 18 & $17,0 \%$ \\
\hline Universidade de São Paulo de Ribeirão Preto & SP & 8 & $7,5 \%$ \\
\hline Universidade Estadual Paulista “Julio Mesquita Neto" & SP & 1 & $0,9 \%$ \\
\hline Universidade Federal de São Paulo & $\mathrm{SP}$ & 1 & $0,9 \%$ \\
\hline Universidade Municipal de São Caetano do Sul & SP & 1 & $0,9 \%$ \\
\hline Universidade Presbiteriana Mackenzie & SP & 3 & $2,8 \%$ \\
\hline Universidade del Valle (Colômbia) & Colômbia & 1 & $0,9 \%$ \\
\hline Total & & 102 & $100 \%$ \\
\hline
\end{tabular}

